\title{
Growth of Mediastinal Bronchial Artery Aneurysms in a Patient With Bronchiectasis
}

\author{
Yoshimi Noda $^{\text {a }}$, Seigo Minami ${ }^{\text {a, b, d }}$, Yoshiki Nishida ${ }^{\mathrm{c}}$, Kiyoshi Komuta ${ }^{\mathrm{a}, \mathrm{b}}$
}

\begin{abstract}
Mediastinal bronchial artery aneurysm (BAA) is a rare and lifethreatening disease. We experienced the first case confirming that, despite only slight deterioration of bronchiectasis, mediastinal BAAs expanded remarkably. A 61-year-old female was hospitalized because of hemoptysis. Her respiratory condition required intubated mechanical ventilation. Bronchial artery angiography unexpectedly found unruptured mediastinal BAAs. These BAAs were enlarged compared with those seen in a computed tomography (CT) taken 5 years before. We successfully embolized both BAAs and peripheral arteries. Mediastinal BAAs are capable of increasing more rapidly than a slightly deteriorated underlying bronchiectasis. For rapid and accurate diagnosis of BAA, bronchial artery angiography is important.
\end{abstract}

Keywords: Mediastinal bronchial artery aneurysm; Bronchiectasis; Bronchial artery embolization; Hemoptysis; Selective bronchial artery angiography; Enhanced CT scan

\section{Introduction}

Mediastinal bronchial artery aneurysm (BAA) is a rare and life-threating disease. This type of aneurysm was observed in less than $1 \%$ of all cases of bronchial arteriography [1]. This disease is classified as follows: congenital or acquired, single or multiple and intrapulmonary or mediastinal. BAA presents various symptoms, depending on its location. Hemoptysis is common in an intrapulmonary BAA, while a mediastinal BAA causes acute thoracic pain, hemothorax, hemomediastinum, superior vena cava obstruction, dysphagia and Horner syndrome due to its extrinsic compression or rupture [2-4].

Manuscript submitted August 16, 2019, accepted August 26, 2019

aDepartment of Respiratory Medicine, Osaka Police Hospital, 10-31 Kitayama-cho, Tennoji-ku, Osaka-City, Osaka 543-0035, Japan

${ }^{b}$ Department of Respiratory Medicine, Daini Osaka Police Hospital, 2-6-40 Karasugatsuji, Tennoji-ku, Osaka, 543-8922, Japan

'Department of Radiology, Osaka Police Hospital, 10-31 Kitayama-cho, Tennoji-ku, Osaka-City, Osaka 543-0035, Japan

${ }^{\mathrm{d} C}$ Corresponding Author: Seigo Minami, Department of Respiratory Medicine, Osaka Police Hospital, 10-31 Kitayama-cho, Tennoji-ku, Osaka-City, Osaka 543-0035, Japan. Email: seigominami@oph.gr.jp

doi: https://doi.org/10.14740/jmc3359
Mediastinal BAAs occasionally remain asymptomatic and are accidentally detected on radiologic examination. The etiology of mediastinal BAA still remains unclear.

We report a rare case of growing multiple mediastinal BAAs, which had been associated with slightly deteriorated bronchiectasis.

\section{Case Report}

A 61-year-old woman was hospitalized because of hemoptysis of approximately $150 \mathrm{~mL} /$ day. Five years prior to this admission, she had been admitted to our hospital because of hemoptysis of $150 \mathrm{~mL} /$ day for two consecutive days. At that time, hemoptysis spontaneously stopped. We had not examined her since the previous discharge. On this admission, her respiratory rate and pulse oximetry on room air were 21 breaths $/$ min and $90 \%$, respectively. Seven hours after the admission, her respiratory condition suddenly deteriorated. Oxygen supply of $10 \mathrm{~L} / \mathrm{min}$ from a mask with reservoir bag was insufficient to maintain her respiratory condition. She was immediately intubated. Plain chest computed tomography (CT) scan revealed bronchiectasis of the right middle lobe and both lower lobes (Fig. 1a). Compared with 5 years before (Fig. 1b), bronchiectasis was deteriorated slightly in the right middle and both lower lobes. Bronchoscopy identified hemoptysis from the right lower lobes. No malignant tumor was detected by these examinations. Thus, we suspected the bronchiectasis as the cause of the hemoptysis. Subsequent bronchial arterial angiography unexpectedly found two unruptured mediastinal BAAs arising from the right bronchial artery, as well as a slight hemorrhage from the peripheral arteries of these BAAs (Fig. 2). We were concerned about rapidly increasing pressure to the BAAs and rupturing them if we were to embolize only the peripheral arteries. Thus, we had to embolize both the mediastinal BAAs and peripheral arteries at one stage. We suspended the bronchial artery embolization (BAE) because of a lack of preparation for BAE of the unexpected BAAs. Thereafter, an enhanced CT scan revealed that these aneurysms were enlarged (Fig. 3a) compared with those seen in the past CT (Fig. 3b). Five years before, the aneurysms were so small that we did not notice them (Fig. 3b). On the ninth hospital day, we tried the BAE again. First, we occluded the branches of the most distal and largest aneurysm with n-butyl-2-cianoacrylate, lipiodol and nine coils (five Trufill $3 \times 20 \mathrm{~mm}$, one Trufill $4 \times 30 \mathrm{~mm}$ and three Deltafill $7 \times 33 \mathrm{~cm}$ ). Second, we embolized the most proxi- 


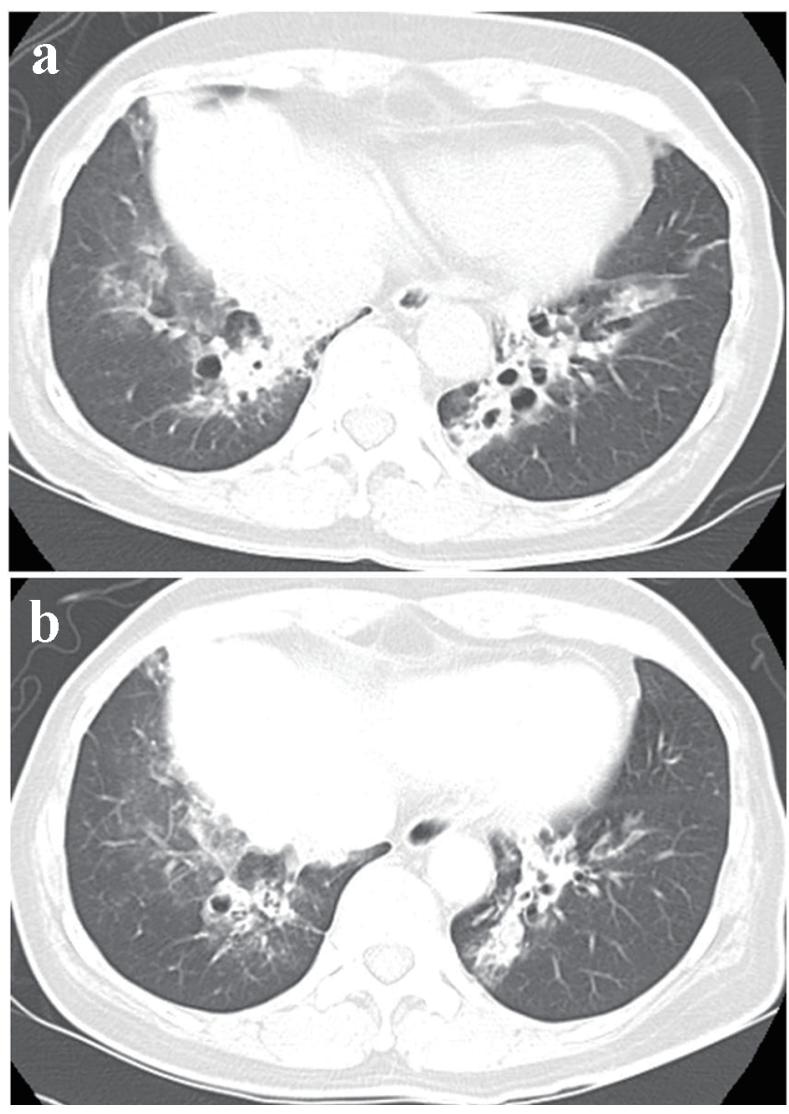

Figure 1. Bronchiectasis (a) at this admission and (b) 5 years ago.

mal and second-largest aneurysm with eight coils (two Micrusframe S $13 \times 43 \mathrm{~cm}$, one Micrusframe $\mathrm{C} 9 \times 22 \mathrm{~cm}$, one Micrusframe C $5 \times 12 \mathrm{~cm}$, two Deltafill $12 \times 42 \mathrm{~cm}$ and two Deltafill $10 \times 40 \mathrm{~cm}$ ). Other aneurysms were so small that we did not treat them at that time. Finally, we confirmed the disappearance of blood flow of the bronchial arteries feeding

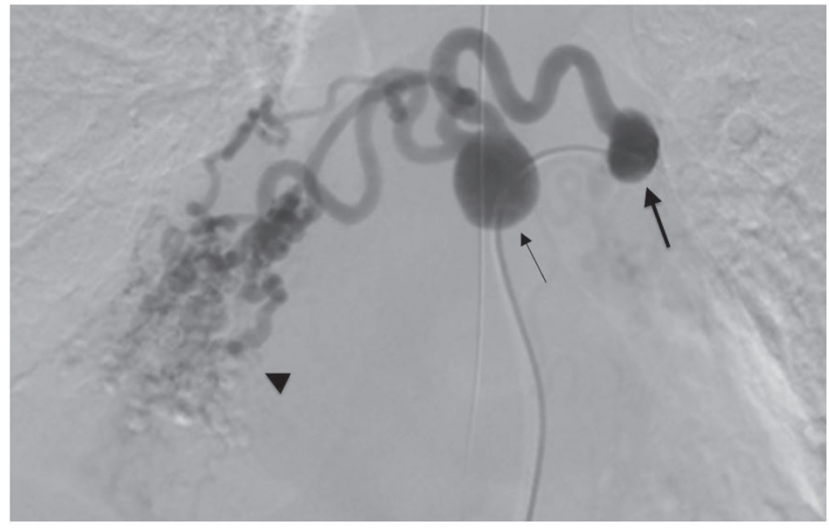

Figure 2. Bronchial arteriography showed two mediastinal bronchial artery aneurysms (arrows) and peripheral arterial dilatation (arrowhead). The proximal bronchial artery aneurysm (thick arrow) arose from the left side of the descending aorta. The bronchial artery crossed in front of the aorta and led to the distal bronchial artery aneurysm (thin arrow). The artery flowed into the right lower lobe of the lung.
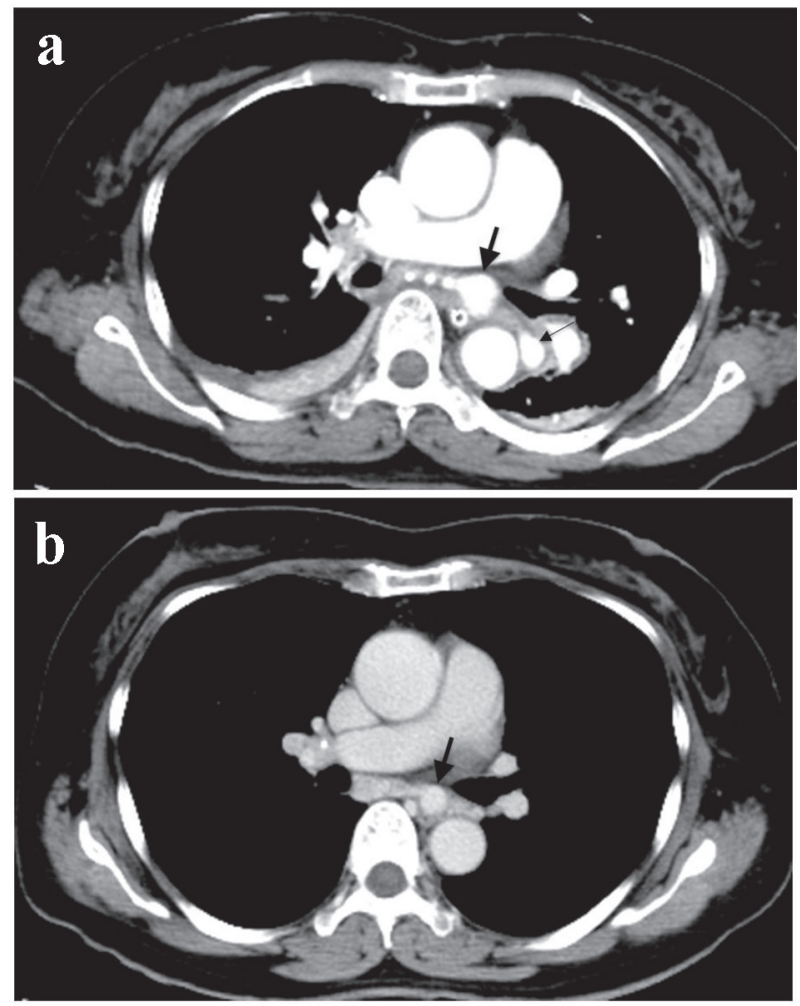

Figure 3. Contrast-enhanced CT scans (a) at this admission and (b) 5 years ago. Mediastinal aneurysm (thick arrow) enlarged during the past 5 years and a new mediastinal aneurysm arose (thin arrow).

the lower lobe and aneurysms (Fig. 4). After the BAEs, her respiratory conditions and hemoptysis improved rapidly. She was extubated and discharged on the 10th and 23th hospital days, respectively. One month after the discharge, enhanced CT scan confirmed no blood flow to the arteries and BAAs. Thereafter, we did not follow up with her because she transferred to another hospital.

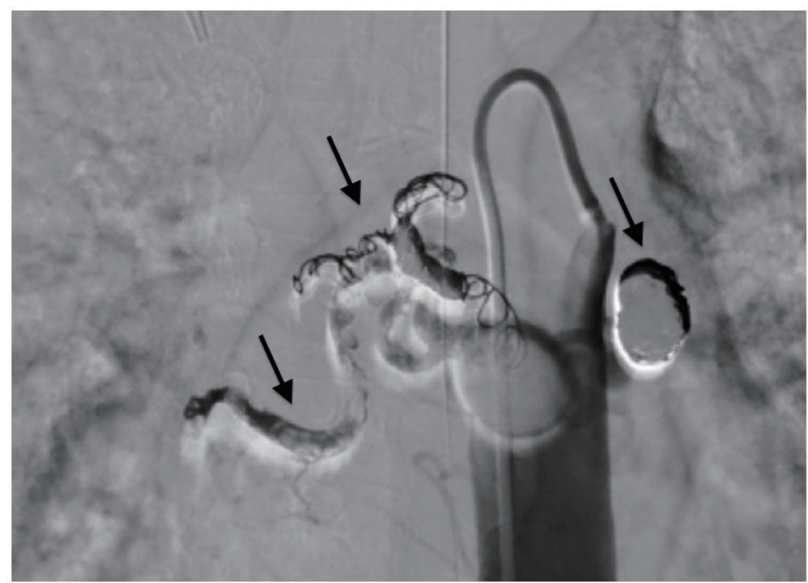

Figure 4. Bronchial arteriography after bronchial arterial embolization. Coils (arrows) occluded two aneurysms and peripheral arteries feeding the right lower lobe. The blood flow into the aneurysms and peripheral arteries disappeared. 


\section{Discussion}

This case was interesting in that, despite only slight deterioration of bronchiectasis, mediastinal BAAs are capable of expanding remarkably. Selective bronchial artery angiography was more sensitive than an enhanced CT for assessing mediastinal BAA.

First, underlying mediastinal BAAs may steadily enlarge even if bronchiectasis appears to be relatively stable. In our case, the CT scan found that, during the previous 5 years, the mediastinal BAAs obviously increased in size while the bronchiectasis slightly worsened. A BAA is acquired as a result of atherosclerosis, inflammatory lung disease (such as bronchiectasis and tuberculosis), systemic disease, or trauma $[5,6]$. In these diseases, increased blood flow and pressure in the bronchial arteries cause weakness or injury to the vessel wall [2, 4, 7-9]. Especially, chronic inflammatory disorders release angiogenic growth factors, which lead to neovascularization, vascular remodeling and increased collateral supply from the nearby systemic arteries [10]. In contrast to the past cases in which BAA caused hemoptysis [1], peripheral bronchial arteries of the BAAs were the origin of hemoptysis in our case. Blood flow and pressure might be increased even in these peripheral arteries.

Second, selective bronchial artery angiography is necessary to detect hidden mediastinal BAAs. In some past cases, contrast-enhanced CT was useful as a diagnostic tool for BAA $[1,8]$. However, in our case, the BAA seen 5 years previously was still too small to be detected only by contrast-enhanced CT scan. The selective arterial angiography at this time easily determined that the BAA was not ruptured and that the peripheral bronchial arteries were the cause of hemorrhage. Thus, for patients with hemoptysis, we should not hesitate to perform angiography, which is the most reliable method for the definite diagnosis of BAAs.

More importantly, BAA rupture causes life-threatening hemorrhage. The aneurysm diameter is not an independent risk factor $[1,2,6,8,10,11]$. Thus, BAAs should be treated as soon they are detected. Nowadays, BAE is increasingly chosen as a treatment method because it is less invasive and requires a shorter hospital stay. In cases of severe complications, such as BAA rupture or technical difficulties, surgical extirpation is an alternative measure.

In conclusion, mediastinal BAAs are capable of increasing more remarkably than a slightly deteriorated underlying bronchiectasis. Hemoptysis is caused by not only BAA but also peripheral arteries. Selective bronchial artery angiography, which is more useful than an enhanced CT for detecting BAA, should be conducted for earlier diagnosis.

\section{Acknowledgments}

None to declare.

\section{Financial Disclosure}

None to declare.

\section{Conflict of Interest}

The authors declare that they have no conflict of interest.

\section{Informed Consent}

The submission and publication of this case report were approved by the Osaka Police Hospital Ethics Committee, because the patient and her relatives were missing and we could not obtain the written informed consent from them.

\section{Author Contributions}

Yoshimi Noda is the first author and wrote the manuscript. All authors were involved in patient treatment and management. Yoshiki Nishida performed angiography. Seigo Minami supervised the writing critically. Kiyoshi Komuta supervised all aspects of this case. All authors read and approved the final manuscript.

\section{References}

1. Kalangos A, Khatchatourian G, Panos A, Faidutti B. Ruptured mediastinal bronchial artery aneurysm: a dilemma of diagnosis and therapeutic approach. J Thorac Cardiovasc Surg. 1997;114(5):853-856.

2. Bak SH, Han H. Diagnosis of bronchial artery aneurysm by computed tomography: a case report. Radiol Case Rep. 2017;12(3):455-459.

3. Kim HJ, Son HS, Cho SB, Kim KT. Development of a life-threatening mediastinal hematoma from a ruptured bronchial artery aneurysm. J Thorac Cardiovasc Surg. 2013;146(5):e47-49.

4. Tanaka K, Ihaya A, Horiuci T, Morioka K, Kimura T, Uesaka T, Sasaki M, et al. Giant mediastinal bronchial artery aneurysm mimicking benign esophageal tumor: a case report and review of 26 cases from literature. J Vasc Surg. 2003;38(5):1125-1129.

5. Hoffmann V, Ysebaert D, De Schepper A, Colpaert C, Jorens $\mathrm{P}$. Acute superior vena cava obstruction after rupture of a bronchial artery aneurysm. Chest. 1996;110(5):13561358.

6. Mizuguchi S, Inoue K, Kida A, Isota M, Hige K, Aoyama T, Ishikawa T. Ruptured bronchial artery aneurysm associated with bronchiectasis: a case report. Ann Thorac Cardiovasc Surg. 2009;15(2):115-118.

7. Hoshi S, Kureya K, Satou K, Fujimoto H, Matsushita H. A case of mediastina bronchial artery aneurysm complicate with bronchiectasis. J Japan Society Resp Endoscopy. 2014;36(5):509-513.

8. Sano M, Kani H. A case of ruptured mediastinal bronchial artery aneurysm. J Japan Society Resp Endoscopy. 2011;33(3):177-181.

9. Katsuda R, Mochizuki Y, Nakahara Y, Kawamura T, Sasaki S, Mayumi T. [A case of ruptured bronchial artery 
aneurysm with hemothorax and mediastinal hematoma]. Nihon Kokyuki Gakkai Zasshi. 2009;47(10):895-899.

10. Salamone I, Cavallaro M, Visalli C, Velo M, Barbaro U, Galletta K, Ando F. Embolization of a bronchial artery aneurysm in a Chronic Obstructive Pulmonary Disease
(COPD) patient with non-massive hemoptysis. Pol J Radiol. 2017;82:174-178.

11. Mahmood RD, Chen ZY, Low TB, Ng KS. A rare case of multiple bronchial artery aneurysms associated with a double aortic arch. Singapore Med J. 2015;56(3):e42-45. 